\title{
Endemic Babesiosis in Another Eastern State: New Jersey
}

\author{
Barbara L. Herwaldt, ${ }^{\star}$ Paul C. McGovern, $†$ Michal P. Gerwel,‡ Rachael M. Easton, $†$ and Rob Roy MacGregor†
}

In the United States, most reported cases of babesiosis have been caused by Babesia microti and acquired in the northeast. Although three cases of babesiosis acquired in New Jersey were recently described by others, babesiosis has not been widely known to be endemic in New Jersey. We describe a case of babesiosis acquired in New Jersey in 1999 in an otherwise healthy 53-year-old woman who developed life-threatening disease. We also provide composite data on 40 cases of babesiosis acquired from 1993 through 2001 in New Jersey. The 40 cases include the one we describe, the three cases previously described, and 36 other cases reported to public health agencies. The 40 cases were acquired in eight (38.1\%) of the 21 counties in the state. Babesiosis, a potentially serious zoonosis, is endemic in New Jersey and should be considered in the differential diagnosis of patients with fever and hemolytic anemia, particularly in the spring, summer, and early fall.

I $\mathrm{n}$ the United States, most of the hundreds of reported cases of babesiosis have been caused by Babesia microti, a parasite of small mammals transmitted by Ixodes scapularis (deer ticks); these ticks also transmit Borrelia burgdorferi and Anaplasma (Ehrlichia) phagocytophila. Most reported cases of babesiosis have been acquired in the northeast, specifically in New York, Massachusetts, Connecticut, and Rhode Island. Another focus of B. microti infection is in Wisconsin and Minnesota (1).

Although three cases of babesiosis acquired in New Jersey in 1998 were described by Eskow et al. (2), babesiosis has not been widely known to be endemic in New Jersey. Of interest, the index case-patient who acquired B. microti infection in the northeast (on Nantucket Island in 1969) actually was hospitalized in New Jersey (3). We describe a case of babesiosis acquired in New Jersey in 1999 and provide composite data that include this case, the three cases previously reported by Eskow et al. (2), and 36 other cases acquired in New Jersey from 1993 through 2001. Our data strengthen the conclusion that babesiosis is endemic in New Jersey.

\section{Methods}

\section{Case Detection and Definition}

We learned of additional babesiosis cases because they were reported to the New Jersey Department of Health and

${ }^{*}$ Centers for Disease Control and Prevention, Atlanta, Georgia, USA; †University of Pennsylvania Health System, Philadelphia, Pennsylvania, USA; and $\ddagger$ New Jersey Department of Health and Senior Services, Trenton, New Jersey, USA
Senior Services or because health-care providers contacted the Centers for Disease Control and Prevention (CDC) about the diagnosis or treatment of babesiosis. Although babesiosis is not a nationally notifiable disease, some states have made cases of babesiosis reportable. Cases became reportable in New Jersey in 1985; however, reporting was discontinued in 1990 because no cases had been reported. Reporting was reinstated in 1995, and 1997 was the first year in which cases were reported to the health department.

We defined a case of babesiosis as an infection occurring in a symptomatic person whose illness was consistent with babesiosis, most likely was acquired in New Jersey, and most likely resulted from a tick bite rather than a blood transfusion. In addition, supporting laboratory data had to be provided and include at least one of the following: identification by light microscopy of intraerythrocytic Babesia parasites in a peripheral blood smear, isolation of the parasite from a whole blood specimen (by inoculating hamsters [Mesocricetus auratus] intraperitoneally and examining smears of blood obtained by tail snip, weekly for up to 2 months), demonstration of B. microti DNA in a whole blood specimen by polymerase chain reaction (PCR) analysis at a reference laboratory, or demonstration of a Babesia-specific antibody titer of at least 1:256 with an indirect fluorescent antibody assay for total immunoglobulin (Ig) or IgG. If only serologic data met the diagnostic criteria, the case was considered probable rather than confirmed.

\section{Case Report}

A previously healthy 53-year-old woman was admitted to a community hospital on June 24, 1999, because she had had 1 week of fever $\left(38.9^{\circ} \mathrm{C}-39.4^{\circ} \mathrm{C}\right)$, rigors, a nonproductive cough, an occipital headache, and increasing malaise. Three days before her hospitalization, she started therapy with cefuroxime axetil for presumed bronchitis but did not improve. She had a $>50$ pack-year history of smoking and drank two to three beers per day. She lived in Burlington County (Figure 1) in southcentral New Jersey and had not traveled outside the county recently. Although she did not recall recent exposure to deer ticks, she occasionally had seen deer in her backyard and she gardened frequently.

On admission to the hospital, she had a temperature of $39.2^{\circ} \mathrm{C}$, a blood pressure level in the $80 / 60 \mathrm{~mm} \mathrm{Hg}$ range, and otherwise unremarkable results on physical examination. She was anemic and thrombocytopenic, with elevated total bilirubin and lactate dehydrogenase values (Table). On the basis of a blood smear from June 24, which showed intraerythrocytic ring forms in approximately $5 \%$ of the erythrocytes on her 


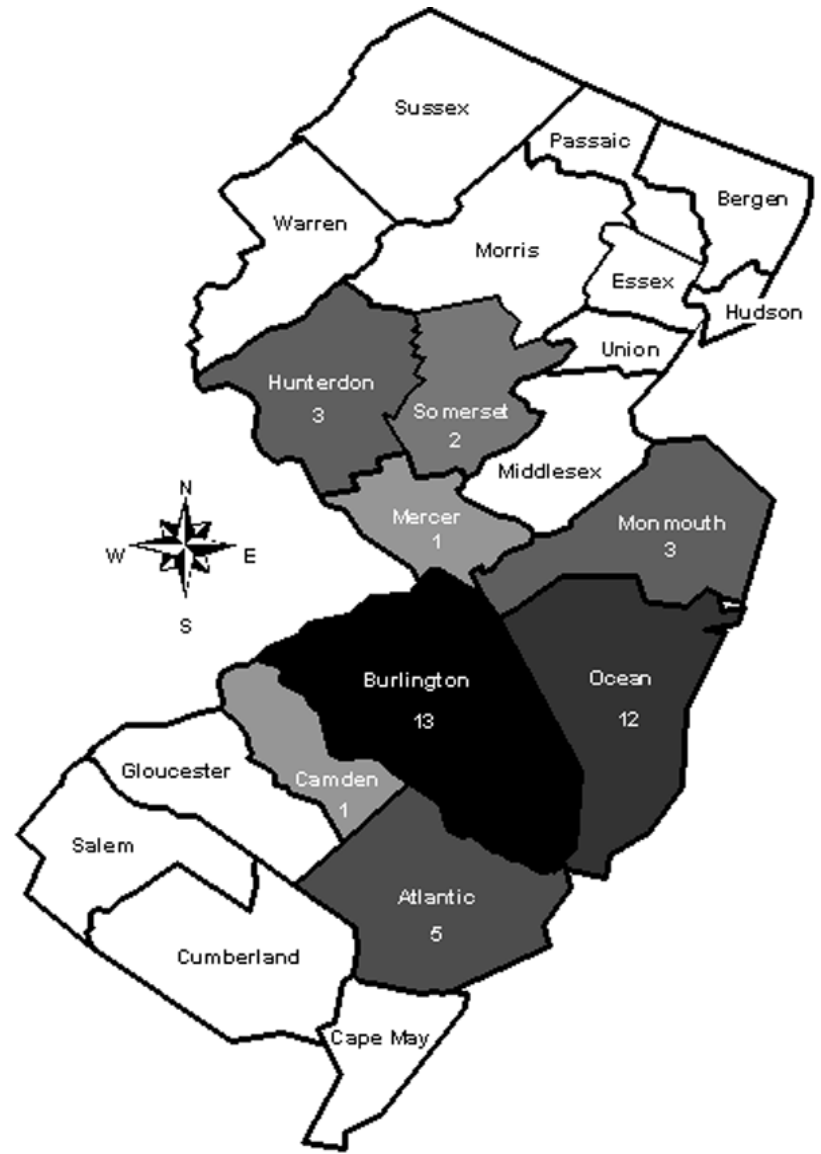

Figure 1. Map of New Jersey showing its 21 counties. The eight counties in which reported cases of babesiosis were acquired from 1993 through 2001 are shaded in gray (the darker the gray, the more cases). The number of cases reported per county is shown under the name of the county.

peripheral blood smear, treatment for babesiosis was begun on June 25. The treatment included intravenous clindamycin, 900 $\mathrm{mg}$ three times a day, and oral quinine, $650 \mathrm{mg}$ three times a day; and she was transfused with two units of packed erythrocytes. Also on June 25, hypoxic respiratory failure developed, and she was intubated. A chest radiograph showed diffuse alveolar infiltrates, which were attributed to the adult respiratory distress syndrome (ARDS).

On June 28, she was transferred to the Hospital of the University of Pennsylvania. When admitted, her blood pressure was $84 / 52 \mathrm{~mm} \mathrm{Hg}$, despite therapy with dopamine. She continued therapy for babesiosis for a total of 15 days (the dose of clindamycin was decreased to $600 \mathrm{mg}$ three times a day on June 28). Although the level of parasitemia had decreased to $0.3 \%$ by June 29 , she had ongoing hemolysis and received six more units of packed erythrocytes during her hospital stay (Table). No parasites were noted on a blood smear on July 9, the last day of antibabesial therapy. She had been successfully weaned from inotropic blood-pressure support on July 2 and underwent extubation on July 4.

Additional laboratory testing at CDC provided further evidence that she was infected with $B$. microti. Serum specimens assayed in parallel, in serial fourfold dilutions, by indirect flu- orescent antibody testing for antibody to B. microti (4), had titers of 1:1,024 (June 30, 1999) and 1:16 (July 16, 2000). In addition, PCR analysis of whole blood from June 30, 1999, by using B. microti-specific primers (5), confirmed she was infected with $B$. microti. Serologic testing performed at the Hospital of the University of Pennsylvania by enzyme immunoassay for antibody to Borr. burgdorferi was negative.

Complications during her hospitalization unrelated to babesiosis included nosocomial pneumonia, acute tubular necrosis from hypoperfusion, bilateral deep venous thromboses, pulmonary embolism, and thrombocytopenia temporally associated with the initiation of heparin therapy (Table). On July 23, after 30 days in the hospital, she was sent home. She was continuing to do well as of October 2002.

\section{Composite Data}

The 40 cases in our analyses include the case described above, the three cases previously described by Eskow et al. (2), and 36 other cases. We did not include six other reported tick-borne cases that occurred in New Jersey residents, because the laboratory data did not meet our criteria or information about the probable state in which infection was acquired was not known or provided.

The number of reported cases of babesiosis increased over time (Figure 2); 28 (70.0\%) of the 40 cases occurred in 2000 or 2001 . The 40 cases were acquired in eight $(38.1 \%)$ of the state's 21 counties (Figure 1). Burlington County, on the inner coastal plain, and Ocean County, on the outer coastal plain, which are neighboring counties in southcentral New Jersey, accounted for $25(62.5 \%)$ of the 40 cases; these two counties are the 7th (Ocean) and 10th (Burlington) most populous counties in the state. None of the cases were acquired in the northernmost or southernmost counties of New Jersey.

Most of the cases were in elderly persons (median age, 67 years; range, 11-87 years). Over half of the cases (22 [55.0\%]) were in male patients. The median date of diagnosis was July 20 (range, June 10-September 9; $\mathrm{n}=36$ ). Two patients (5.0\%) died: an 86-year-old man with multisystem organ failure and an 80-year-old man with ARDS. The patient whose case we described here also developed ARDS.

The following information about the patients was not collected systematically. However, three patients were reported to be asplenic, 18 to have recalled tick bites, 34 to have been hospitalized, and three to have had Lyme disease (no details available). Underlying conditions included HIV infection in one patient, who had a CD4 count of 50; diabetes in five patients; a history of breast or prostate cancer in three patients (no details available); and a condition that led to chemotherapy in one person (no details available).

Various types of laboratory tests were used to diagnose the 40 babesiosis cases. Not all patients were tested with the same methods. However, 34 patients had positive blood smears; for 27 of these patients, the positive smear was the only laboratory result that met our diagnostic criteria. All three cases reported by Eskow et al. were in patients who had negative blood 
RESEARCH

Table. Clinical data on selected dates for a patient who acquired babesiosis in New Jersey

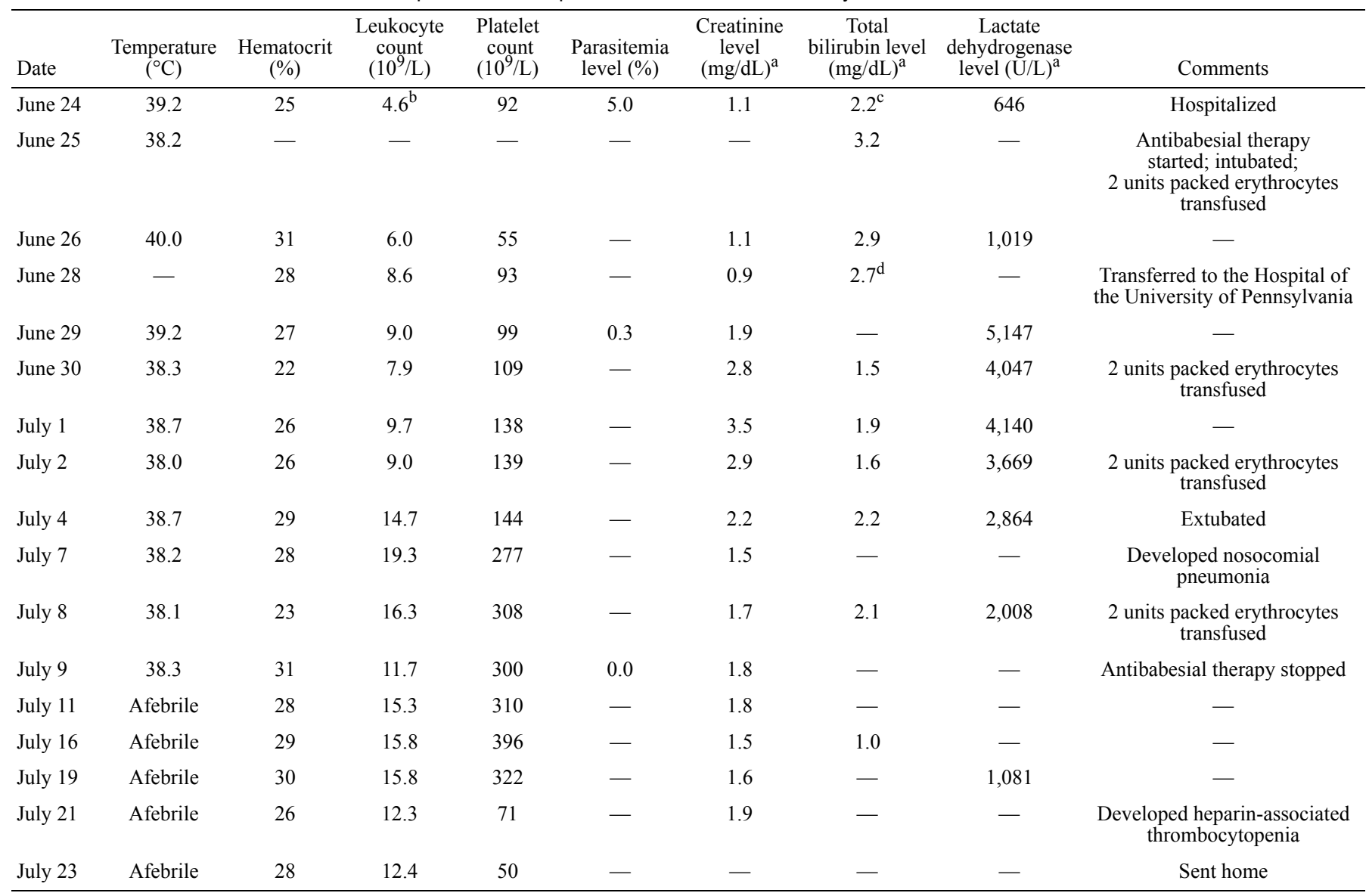

${ }^{a}$ Normal ranges for community hospital (June 24-June 27): creatinine level, $0.6-1.3 \mathrm{mg} / \mathrm{dL}$; total bilirubin level, $0.2-1.0 \mathrm{mg} / \mathrm{dL}$; indirect bilirubin level, $0.2-1.2 \mathrm{mg} / \mathrm{dL}$; lactate dehydrogenase level, 91-180 U/L. Normal ranges for the Hospital of the University of Pennsylvania: creatinine level, $0.6-1.0 \mathrm{mg} / \mathrm{dL}$; total bilirubin level, $0.0-1.2 \mathrm{mg} / \mathrm{dL}$; indirect bilirubin level, 0.0-1.2 mg/dL; lactate dehydrogenase level, 313-618 U/L.

$\mathrm{b}_{25} \%$ segmented neutrophils, $33 \%$ band forms, $22 \%$ lymphocytes, $2 \%$ atypical lymphocytes, $8 \%$ monocytes.

${ }^{\mathrm{c}}$ Indirect bilirubin level, $1.4 \mathrm{mg} / \mathrm{dL}$.

${ }^{\mathrm{d}}$ Indirect bilirubin level, $2.1 \mathrm{mg} / \mathrm{dL}$; serum haptoglobin level, $<38 \mathrm{mg} / \mathrm{dL}$ (normal range, $60-160 \mathrm{mg} / \mathrm{dL}$ ); results of direct and indirect Coombs test, negative.

smears (2). One of the two patients who had whole blood inoculated into hamsters had positive results (i.e., the hamsters became parasitemic). Four patients had positive PCR results from a reference laboratory; for one of these patients, these results were the only ones that met our diagnostic criteria. Twelve patients had serologic data that met our criteria; for four patients, the serologic results were the only data that met our diagnostic criteria. These cases were considered probable rather than confirmed. CDC confirmed the diagnosis of Babesia infection in $11(27.5 \%)$ of the 40 cases; specimens from the other 29 case-patients were not sent to CDC.

\section{Discussion}

Our report strengthens the evidence that New Jersey is one of the eastern states in which babesiosis is endemic. In addition, the risk for acquisition of infection is widely distributed in the state. Whether the fact that most of the reported cases occurred in southcentral and northcentral counties reflects the degree of endemicity of babesiosis in various areas of New Jersey is unknown.
The fact that babesiosis is endemic in New Jersey is not surprising, given that Lyme disease, the etiologic agent of which also is transmitted by $I$. scapularis, is highly endemic in New Jersey $(6,7)$ and given the geographic proximity of New Jersey to areas in the northeast where babesiosis is highly endemic. In a 1996 study, of 100 I. scapularis ticks collected in Hunterdon County, New Jersey, 43 were infected with Borr. burgdorferi, 5 were infected with B. microti, and 2 were infected with both organisms (8).

The increase in reported cases of babesiosis, which began in 1998 (Figure 2) and was even more notable in 2000 and 2001, could indicate an increased risk for B. microti infection and illness. If true, possible reasons for the increased risk could include a growing abundance of local $I$. scapularis populations or the introduction of a more virulent strain of B. microti (9). However, the increased numbers of reported cases could simply represent an increased awareness of the disease and increased reporting. Even so, the 40 cases of babesiosis that we tallied probably represent only a fraction of the clinical cases of B. microti infection acquired in New Jersey from 1993 


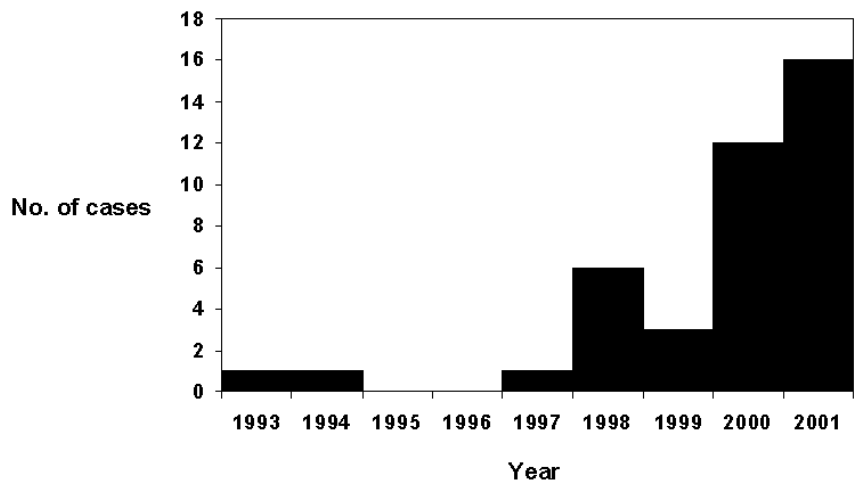

Figure 2. The number of reported cases of babesiosis acquired each year, New Jersey, 1993-2001.

through 2001. Presumably, other symptomatic cases (as well as many more subclinical cases) occurred but were not diagnosed or reported. In fact, several other possible symptomatic cases were reported that we did not count because we received insufficient information about them. Also, as is commonly true for surveillance data, the amount and quality of the information provided to the health department and CDC about the cases varied widely; some of the information might have been inaccurate, and not all of the cases were confirmed by reference laboratories (e.g., not all of the blood smears that were reported as positive were reexamined by a reference laboratory).

The laboratory tests CDC offers for babesiosis, when indicated, include examination of blood smears, hamster inoculation, and PCR (5) for parasitologic diagnosis and an indirect fluorescent antibody assay for total immunoglobulin for serologic diagnosis (4). Using PCR for detection of DNA from Babesia spp. has not yet become a routine diagnostic method, and the analysis should be conducted by experienced reference laboratories.

Immunoblot testing for $\operatorname{IgG}$ and $\operatorname{IgM}$ is investigational. However, an immunoblot test for IgG performed well in a recent evaluation, with a sensitivity of $96 \%$ and a specificity of $99 \%$ (10). A positive serologic result for IgM (11) is insufficient for diagnosis without a positive result for IgG. If the IgM result is positive but the IgG result is negative, a follow-up specimen should be tested. If IgG seroconversion is not noted, the IgM result likely was a false positive. Future serologic testing might involve recombinant and synthetic antigens (12) rather than whole parasites or soluble antigens.

The case we described in detail demonstrates that babesiosis can be life threatening $(1,13,14)$. In fact, two $(5.0 \%)$ of the 40 case-patients died. In the patient we described, the following conditions developed: severe anemia, for which she was transfused with eight units of packed erythrocytes; hypotension that required inotropic support; ARDS, which has previously been reported (13-17); and various nosocomial complications. The fact that she was ill for approximately 1 week before therapy for babesiosis was initiated might have contributed to the severity of her illness. Fortunately, treatment for babesiosis was begun soon after she was hospitalized. Although she was treated successfully with clindamycin and quinine, a recent clinical trial indicated that the combination of azithromycin and atovaquone is also effective (18). However, patients with life-threatening babesiosis were excluded from the study. Severely ill patients, particularly those with high levels of parasitemia (e.g., $\geq 10 \%$ ), may benefit from exchange transfusion $(1,19)$.

In summary, babesiosis, a potentially serious zoonosis, is endemic in New Jersey and should be considered in the differential diagnosis of patients with fever and hemolytic anemia, particularly in the spring, summer, and early fall.

\section{Acknowledgments}

We thank the laboratory staff of the Division of Parasitic Diseases, $\mathrm{CDC}$, and the other laboratorians and physicians who provided data about the cases of babesiosis.

Dr. Herwaldt is a medical epidemiologist in the Division of Parasitic Diseases, Centers for Disease Control and Prevention.

\section{References}

1. Herwaldt BL, Springs FE, Roberts PP, Eberhard ML, Case K, Persing $\mathrm{DH}$, et al. Babesiosis in Wisconsin: a potentially fatal disease. Am J Trop Med Hyg 1995;53:146-51.

2. Eskow ES, Krause PJ, Spielman A, Freeman K, Aslanzadeh J. Southern extension of the range of human babesiosis in the eastern United States. J Clin Microbiol 1999;37:2051-2.

3. Western KA, Benson GD, Gleason NN, Healy GR, Schultz MG. Babesiosis in a Massachusetts resident. N Engl J Med 1970;283:854-6.

4. Chisholm ES, Ruebush TK II, Sulzer AJ, Healy GR. Babesia microti infection in man: evaluation of an indirect immunofluorescent antibody test. Am J Trop Med Hyg 1978;27:14-9.

5. Persing DH, Mathiesen D, Marshall WF, Telford SR, Spielman A, Thomford JW, et al. Detection of Babesia microti by polymerase chain reaction. J Clin Microbiol 1992;30:2097-103.

6. Centers for Disease Control and Prevention. Lyme disease-United States, 1999. MMWR Morb Mortal Wkly Rep 2001;50:181-5.

7. Orloski KA, Campbell GL, Genese CA, Beckley JW, Schriefer ME, Spitalny KC, et al. Emergence of Lyme disease in Hunterdon County, New Jersey, 1993: a case-control study of risk factors and evaluation of reporting patterns. Am J Epidemiol 1998;147:391-7.

8. Varde S, Beckley J, Schwartz I. Prevalence of tick-borne pathogens in Ixodes scapularis in a rural New Jersey county. Emerg Infect Dis 1998;4:97-9.

9. Tsuji M, Wei Q, Zamoto A, Morita C, Arai S, Shiota T, et al. Human babesiosis in Japan: epizootiologic survey of rodent reservoir and isolation of new type of Babesia microti-like parasite. J Clin Microbiol 2001;39:4316-22.

10. Ryan R, Krause PJ, Radolf J, Freeman K, Spielman A, Lenz R, et al. Diagnosis of babesiosis using an immunoblot serologic test. Clin Diag Lab Immunol 2001;8:1177-80.

11. Krause PJ, Ryan R, Telford S III, Persing D, Spielman A. Efficacy of immunoglobulin $\mathrm{M}$ serodiagnostic test for rapid diagnosis of acute babesiosis. J Clin Microbiol 1996;34:2014-6.

12. Lodes MJ, Houghton RL, Bruinsma ES, Mohamath R, Reynolds LD, Benson DR, et al. Serological expression cloning of novel immunoreactive antigens of Babesia microti. Infect Immun 2000;68:2783-90.

13. White DJ, Talarico J, Chang H-G, Birkhead GS, Heimberger T, Morse DL. Human babesiosis in New York State. Review of 139 hospitalized cases and analysis of prognostic factors. Arch Intern Med 1998;158:2149-54. 
14. Hatcher JC, Greenberg PD, Antique J, Jimenez-Lucho VE. Severe babesiosis in Long Island: review of 34 cases and their complications. Clin Infect Dis 2001;32:1117-25.

15. Gordon S, Cordon RA, Mazdzer EJ, Valigorsky JM, Blagg NA, Barnes SJ. Adult respiratory distress syndrome in babesiosis. Chest 1984;86:633-4.

16. Boustani MR, Lepore TJ, Gelfand JA, Lazarus DS. Acute respiratory failure in patients treated for babesiosis. Am J Respir Crit Care Med 1994;149:1689-91.

17. Horowitz ML, Coletta F, Fein AM. Delayed onset adult respiratory distress syndrome in babesiosis. Chest 1994;106:1299-301.
18. Krause PJ, Lepore T, Sikand VK, Gadbaw J, Burke G, Telford SR III, et al. Atovaquone and azithromycin for the treatment of babesiosis. N Engl J Med 2000;343:1454-8.

19. Dorman SE, Cannon ME, Telford SR III, Frank KM, Churchill WH. Fulminant babesiosis treated with clindamycin, quinine, and whole-blood exchange transfusion. Transfusion 2000;40:375-80.

Address for correspondence: Barbara L. Herwaldt, Centers for Disease Control and Prevention, Division of Parasitic Diseases, 4770 Buford Hwy NE, Mailstop F22, Atlanta, GA 30341-3724, USA; fax: 770-488-7761; e-mail: bxh4@cdc.gov
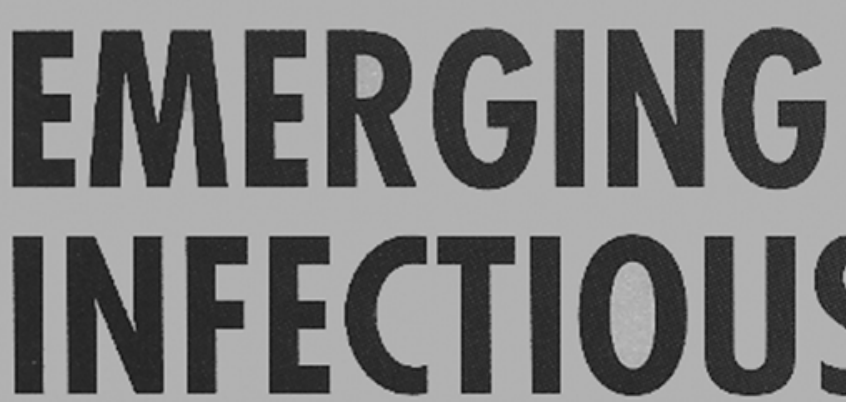

A Peer-Reviewed Journal Tracking and Analyzing Disease Trends
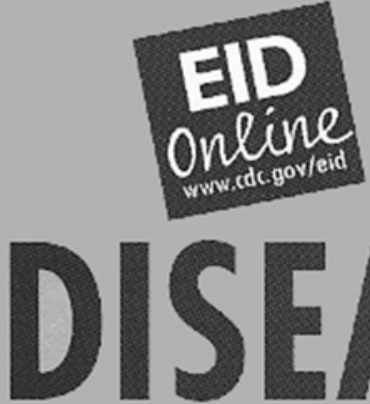

Vol.8, No.4, April 2002

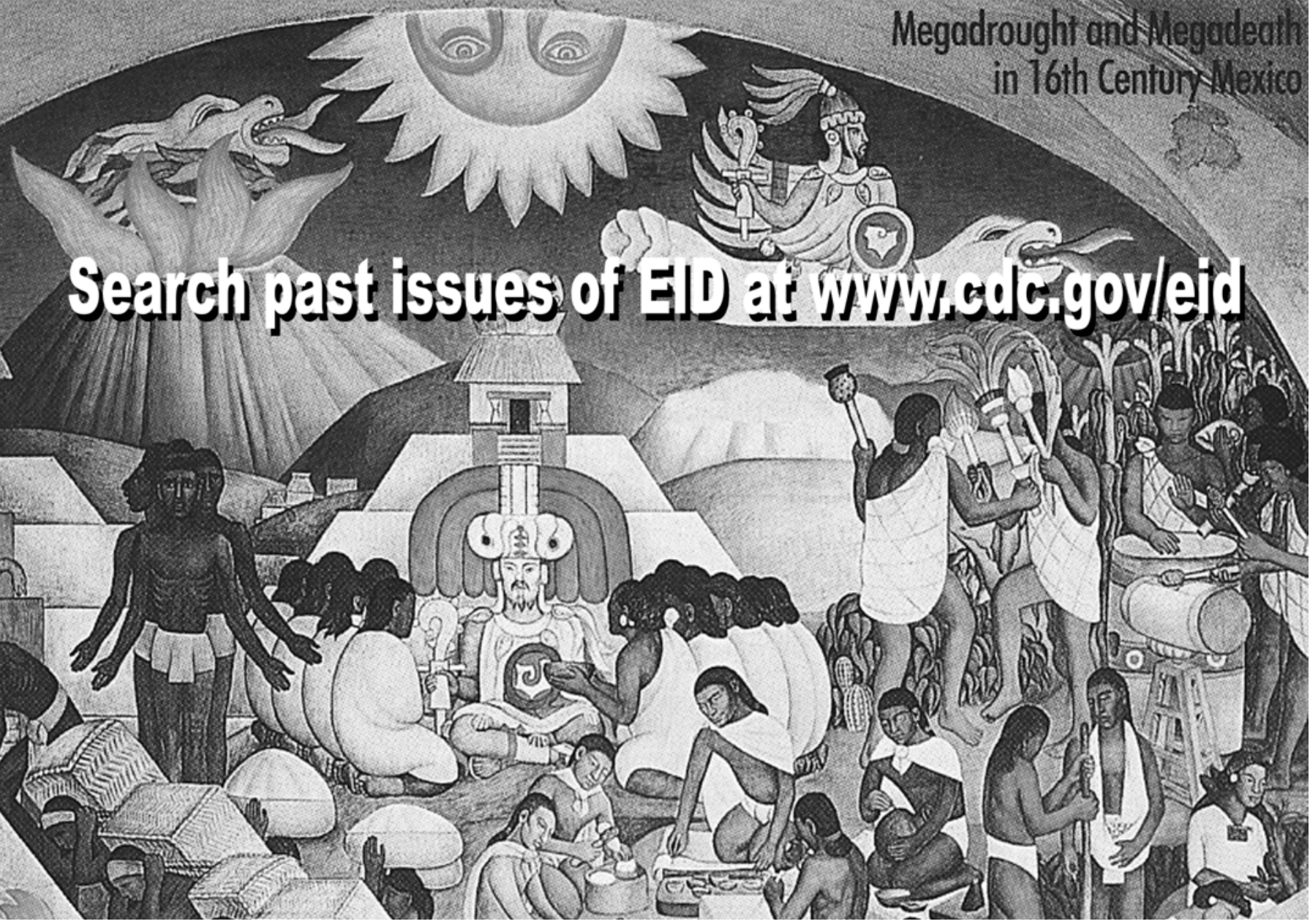

